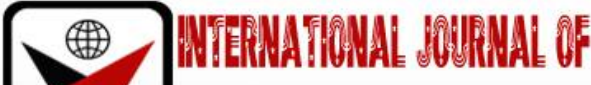

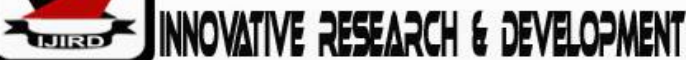

ISSN 2278 - 0211 (Online)

\section{Diagnosing Novel Covid-19 Using Smart Portable Detection Device}

Abdulsalam Auwal Jamilu
Post Graduate Student, Department of Computer Science,
Ahmadu Bello University, Zaria, Nigeria
Abubakar Lawal
Graduate Student, Department of Physics,
Ahmadu Bello University, Zaria, Nigeria
Folorunsho Kudirat Amoke
Graduate Student, Department of Computer Science,
Ahmadu Bello University, Zaria, Nigeria
Ameerah Muhammad Ashir
Graduate Student, Department of Computer Science,
Ahmadu Bello University, Zaria, Nigeria

\begin{abstract}
:
In early December 2019, an outbreak of coronavirus disease (COVID-19) occurred, caused by a novel acute respiratory syndrome (SARS-CoV-2) occurred in Wuhan City, Hubei Province China, the outbreak was declared a Public Health Emergency of International concern by the World Health Organization (W.H.O) on 30 January, 2020. After the outbreak, scanning using thermal infrared thermometers has been used in public places to test the temperature of the body to identify infectious persons in the crowd. This method of prevention is still not enough because it has some weaknesses; it spends a lot of time checking the temperature of each person, it not more intelligent and perhaps the most important thing is that the direct contact of the infected person might result to the dispersal of the disease to the personnel of the screening process and to other people at large. This study proposes the design of a system using a smart device, a programmable micro - controller designed to detect Corona Virus, with less human interactions with the following sensors; optical camera sensor, thermal imaging processing, Bluetooth module, Microphone module and neural processing engine for monitoring the screening process. The device informs authorities the need to acquire data in real time for immediate notification to the threat. The proposed system can be used as a device for healthcare, banking, markets, stores, schools, worship centers, road checkpoints and many more public places. The smart portable device can also be useful for potential travel restrictions, data obtained from the smart portable device can be used to determine the timing of covid-19 vaccine campaigns, collected data from this device can be used for the allocation of medical supplies as it generates real - time data. Finally, this device will help prevent the continuous spread of corona virus, which is increasing every day.
\end{abstract}

Keywords: COVID-19, novel corona virus, smart device, Arduino, healthcare

\section{Introduction}

Corona virus, also known as COVID-19, is a part of the Coronaviridae and Nidovirales family. The family divided into sub-families, Coronavirinae and Torovirinae [1]. COVID-19 is known to be a novel human-infecting betacoronavirus [2]. Phylogenetic analysis of the COVID-19 genome shows that the virus is closely connected (88\% identity) to two batderived SARS-like coronaviruses obtained in 2018 in Eastern China (bat-SL-CoVZXC21 and bat-SL-CoVZC45) and genetically different from SARS-CoV (about 79\% similarity) as well as MERS-CoV[2]. Such findings suggest that bats may be the original host of this VIRUS [2,3]. Person-to - person spread is thought to occur between direct contacts mainly and via respiratory droplets formed when an infected person coughs or sneezes. Pathogenic organisms can be a major route of contamination, as SARS-CoV was already found to survive on surfaces up to 96 hours [4] and other coronaviruses approximately 10 days [5]. A research suggested that the mean incubation period was 5.2 days (95\% confidence interval [95 \% CI] 4.1 to 7.0) [6]. The incubation time was found to be as long as 19 or 24 days [7, 8], even though case definitions rely heavily on a 14-days [9]. It is clear that COVID-19 is a serious disease of international concern. Some estimates have a greater reproductive rate than SARS [10] and much more people were reported to have been infected and to have died from it than SARS [11]. Similar to SARS-CoV and MERS-CoV, disruption of the transmission is considered essential to 
stopping the spread of the disease [12]. Different methods have been introduced in health care settings both at global and at national levels. Worse still, health care settings may be a major source of viral transmission as presented in the SARS framework, the use of triage following correct infection control practices, case isolation and contact tracing are vital to limiting the further spread of the virus in clinics and hospitals [12]. In a community setting, isolation of infected people is the primary means to avoid the transmission. For example, the rapid measures taken by the Chinese health agencies included the isolation of infected persons and the quarantine of suspected people and their near contacts [13]. Also, because there are still conflicting assertions about the animal origins of the virus (i.e. some studies linked the virus to the bat $[14,15]$ whereas others related the virus to the snake [16]), contact with these animal fluids or tissues or consumption of wild caught animals must be avoided. In addition, public awareness of unusual symptoms such as chronic coughing or shortness of breath is critical therefore that they could seek medical care for quick detection of the virus and prevention. In the event of large-scale community transmission, prevention of social gatherings, provisional school closure, close monitoring of symptomatic individuals, requirement of life support (e.g. oxygen, mechanical respirator), home isolation, individual hand hygiene and having to wear of personal protective equipment like face masks must also be enforced [17]. In a wider perspective, the lock-down of Wuhan was one of the immediate actions taken by the Chinese government and hence started to slow the spread of COVID-19 [17]. Air travel should be limited in cases where there is no need for serious medical attention. Setting up a temperatures check or a scan is essential at the airport, borders and other public places, to identify suspicious cases. Continuous preventive measures are essential in the research of this outbreak and providing evidence for future occurrence [17]. Following this introductory section, the rest of the paper is organized into different sections, each focusing on different feature of the research work, below is the summary of the remaining section of the paper; Section I provides the methodology, Section II discuss the conceptual model, Section III entails the results \& discussion and finally section IV is the conclusion followed by references.

\section{Methodology}

This section outlines the working flow of the system and sub-system due to the inter-relationship of the entire application. Arduino Integrated Development Environment 1.8.12 (IDE) is adopted, which is a multi - platform coding environment. It includes many features of the code editor, such as syntax highlighting, easy-indentation, and brace aligning. In addition, the IDE uploaded the code into Arduino board by compiling and uploading programs using the necessary one-click mechanism. It is mainly used for drawing a number of schematics and performing a simulation of realtime circuits that enables humans to gain access during the running phase, thus generating a simulation in real-time [1819]. Normal APIs are created during the Open CV library programming process. Arduino UNO board is used with a Neural Computing Engine powered by an Intel processor. Face detection is achieved using the Cascade Classification algorithm, this is offered by Paul viola and Michael [20]. Recognition of facial expression is of practical significance in medical care using the Convolutional Neural Network (CNN) [21]. Most typical features can be extracted from the human face to allow image data pre-processing with the algorithm [20].

The simulation is done using proteus software and Arduino software. The code is firstly written on the Arduino Integrated Development Environment (IDE), for temperature sensor, sound sensor and the Bluetooth module. The result of the simulation is displayed on the virtual terminal of proteus for viewing. The pin legs of each sensor was declared on the declaration space, then followed by 'void setup' where the input and the output pins of the sensors are setup, and then lastly followed by the 'void loop' where the repetition of the sensor results is carried out, then the code is compiled and uploaded in other to transfer or copy the compiled code to the Arduino board. This is done to test and to confirm that there were no errors, seeing the results on the serial monitor of the Arduino software which is transferred to phone or computer through the Bluetooth module. Simulating using the proteus software confirm the control technique to identify if there are any error. The task of collecting the required data is assigned to the smart device when necessary. Interfacing of a device based on a communication connection is done. This system provides notification when the temperature is higher than normal. The GPS module determines the position of the person after the marking and sends a notification via the internet to the assigned authorities. The micro - controller processor is incorporated with Arduino-UNO via Arduino IDE to perform the coding for compilation of appropriate commands and source code into the processor. Additionally, another segment of the system focuses on the mechanism's output source. The smart portable device was equipped with two different camera kinds, allowing detailed information on face detection and temperature measurements to be collected. Optical camera and infrared thermal camera, provides information on the temperature at which the various points of interest were identified. The thermal camera, sometimes referred to as thermal imaging, thermal imaging, is a device that uses infrared radiation to create an image similar to a conventional camera that uses visible light to produce an image. Thermal camera is used to detect hot bodies and to recognize the high temperature variability compared to other objects within the scanned region. Whenever thermal camera visualizes the body at high temperatures, it creates high infrared spectra intensity. The next section describes the conceptual model.

\section{Conceptual Model}

The essential work flow of the system as shown in Fig. 1 starts by testing the suspected persons using the smart device, if symptoms like high temperature, persistent cough and other related COVID-19 symptoms, then it captures the victim face using Optic and thermal camera embedded in the smart device otherwise it shows a negative result, meaning there are no symptoms related to COVID-19 detected, it then Bproceeds by specifying the GPS location and finally sends notification and GPS location to the monitoring authorities for updates, persons whose result are positive will be physically taken to the nearest Isolation center by trained healthcare personnel, asides detecting persons with COVID-19, 
the device can be used to determine the timing for COVID-19 vaccine campaign, allocation of medical supply can also be at ease since the device generates real-time data.

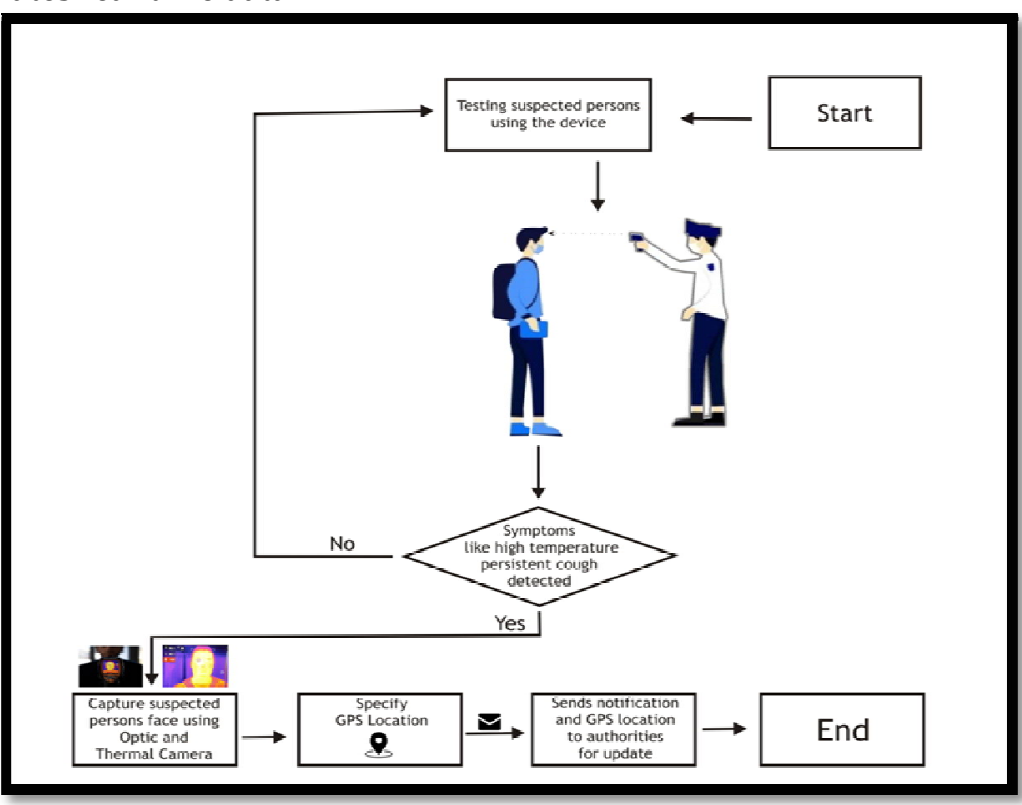

Figure 1: Smart Device Workflow

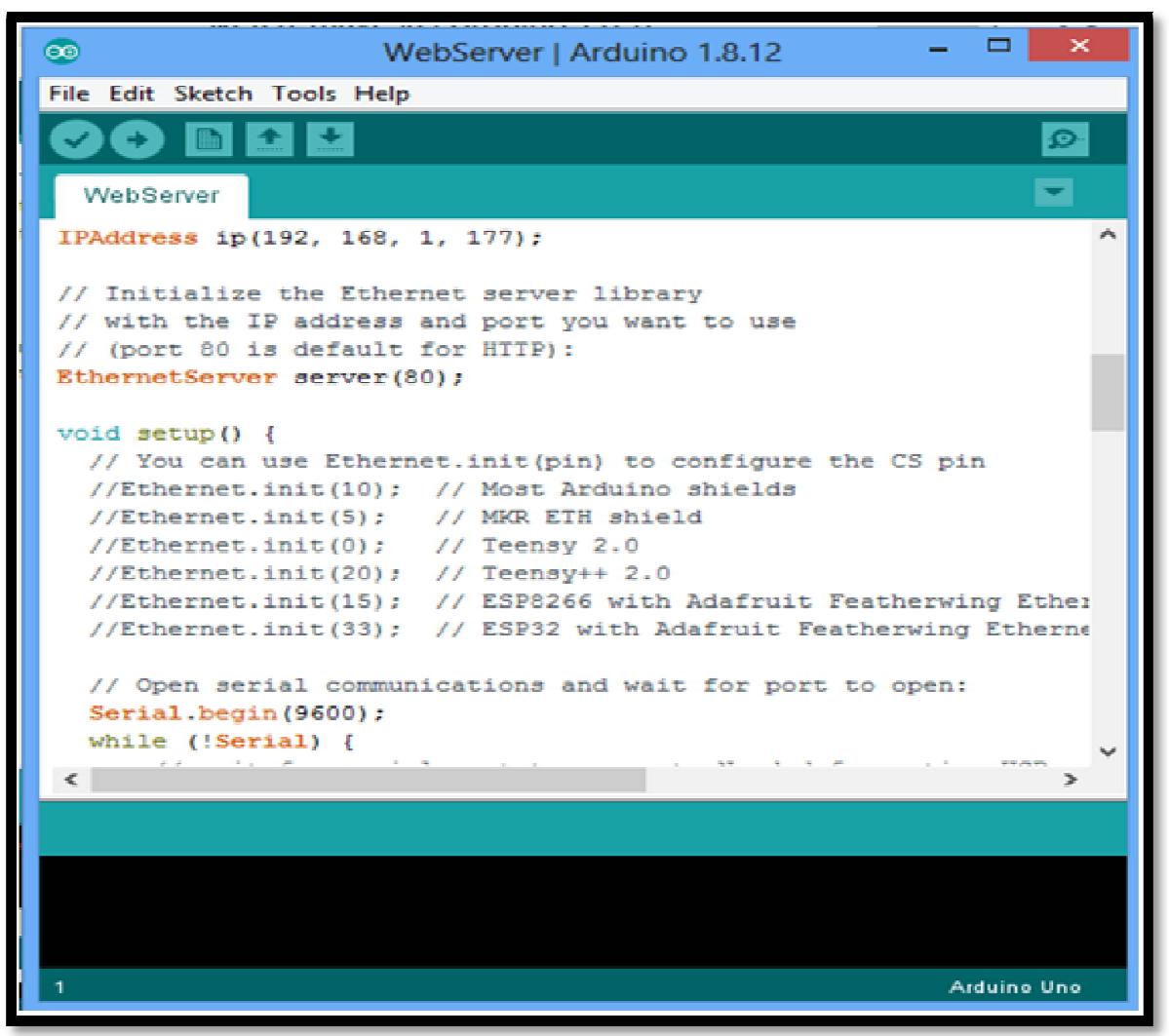

Figure 2: Anduino Coding environment

The device shown in Fig. 3 current research concerns the appropriate form of thermal imaging device for the identification and tracking of body temperature changes and, more precisely, smart camera system could be rapidly implemented and used to visualize high-resolution thermal image for infected persons. The temperature is determined and the device is activated. The intelligent board cooperated into the device, helps individuals with high body temperature to be detected easily, accurately and send real-time report to appropriate agency. This device diagnose disease by screening images and sounds related to persistent coughing which can render diagnostics easier and reduce the chances of making mistakes. The next section explains the results with discussion.

\section{Result and Discussions}

The proposed design performed well at the testing procedure, in order to confirm its feasibility and confirm the Performance of the control technique that has been adopted, Fig. 4 shows the proteus simulation environment. 


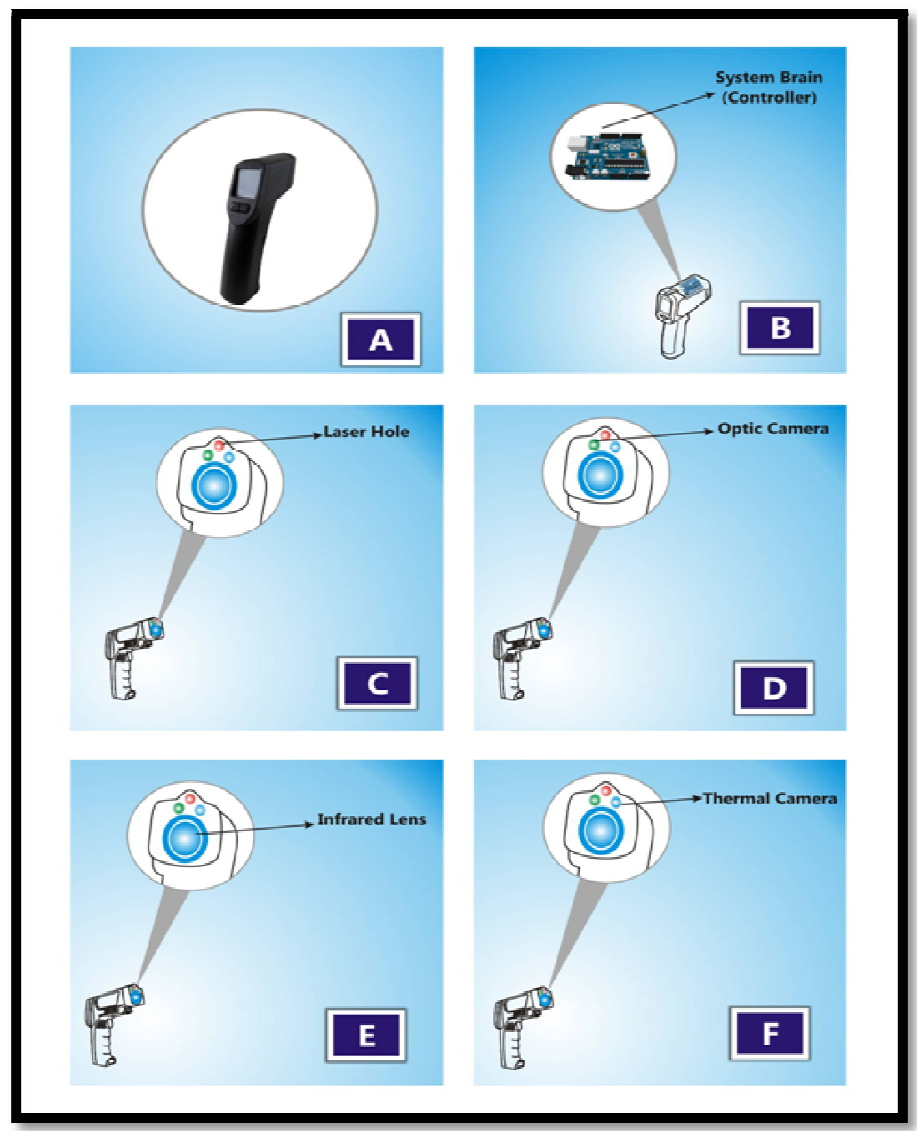
Figure 3: System Components
A. Complete System
B. Controller
C. Laser hole
D. Optic Camera
E. Infrared Lens
F. Thermal Camera

The simulation is attain using the framework Proteus as shown in Fig. 4. In hardware, Arduino is a device consisting of a relatively basic base board with Arduino's Visual Designer (AVR) microcontroller, electronic support and Arduino pin headers (connectors). This is therefore a variety of peripheral boards (shields) that plug in the pin headers to form the design hardware, within AVR, the development of the model consist of stages at which decisions are made on the board Arduino Uno.

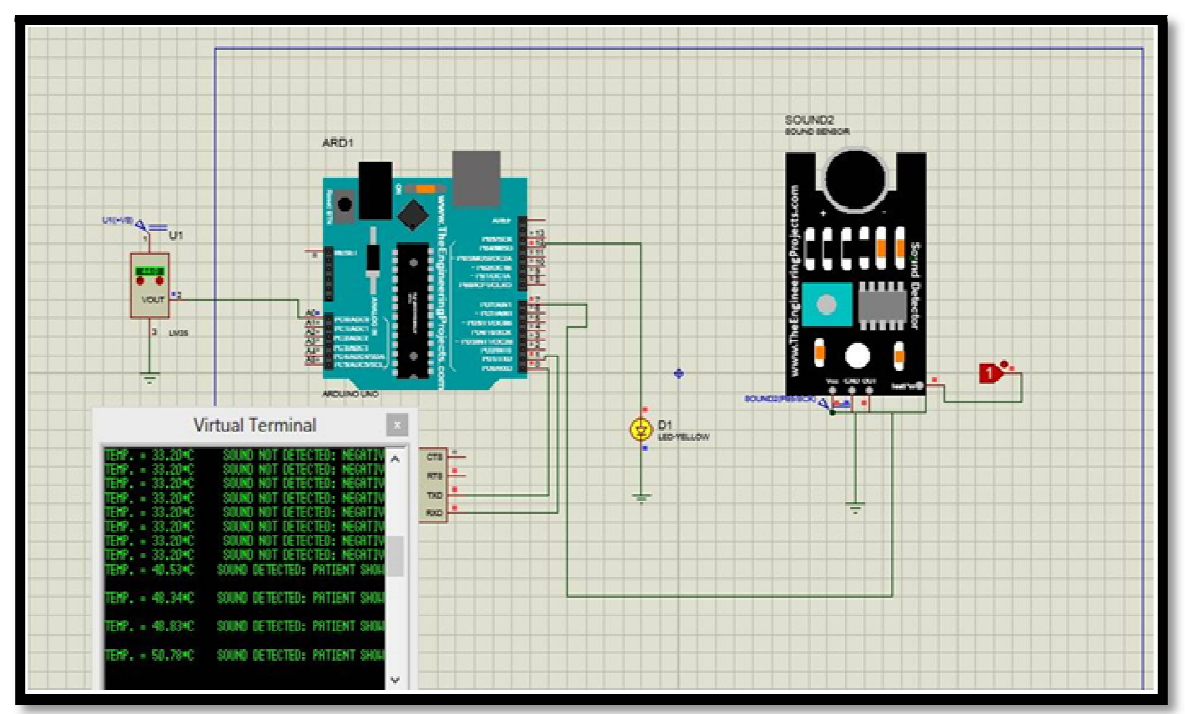

Figure 4: Proteus Simulation Environment

Upon obtaining information, integrating the recognized face, body temperature and GPS location provided by Arduino via consecutive contact, the microcontroller will communicate to it, through the Web (Fig. 2) to provide independent online access to this data. Whenever the thermal camera detects a high-temperature, the system notifies the 
authorities of the threat. The result of the simulation is displayed on the virtual terminal of proteus for viewing as shown in Fig. 4. Around the same time, the device will snap a screenshot and transfer it to the health officer.

\section{Conclusion}

An innovative, early-time coronavirus detection and monitoring system has been developed using a smart device integrated with a thermal imaging system. Smart devices can detect high body temperatures of suspected persons with COVID-19 and send the measured data to be viewed mostly on computer As a result of this pandemic, the spread of coronavirus provides so much attention and knowledge among people. Prompt identification of coronavirus symptoms would be one of the most effective ways to avoid coronavirus spread. As a person's high temperature is among the most common symptoms, a real-time screening system is needed that automatically displays person's temperature and send update to authorities. The diagnosis of the screening process would therefore be less time-consuming and less human interactions that could cause the coronavirus to spread more quickly. This can be concluded that remote sensing procedures, which provide a means of detecting, sensing and tracking coronavirus, offer an remarkable promise and ability to meet the needs of the healthcare system.

\section{References}

i. Burrell C, Howard C, Murphy F: Fenner and White's (2016). Medical Virology 5th Edition. United States: Academic Press;

ii. Lu R, Zhao X, Li J, Niu P, Yang B, Wu H, Wang W, Song H, Huang B, Zhu N et al (2020). Genomic characterisation and epidemiology of 2019 novel coronavirus: implications for virus origins and receptor binding. Lancet.

iii. Zhou P, Yang XL, Wang XG, Hu B, Zhang L, Zhang W, Si HR, Zhu Y, Li B, Huang CL et al (2020). A pneumonia outbreak associated with a new coronavirus of probable bat origin. Nature.

iv. Kramer A, Schwebke I, Kampf G: (2006). How long do nosocomial pathogens persist on inanimate surfaces. A systematic review. BMC Infect Dis, 6:130.

v. Kampf G, Todt D, Pfaender S, Steinmann E (2020). Persistence of coronaviruses on inanimate surfaces and its inactivation with biocidal agents. J Hosp Infect.

vi. Yufika A, Wagner AL, Nawawi Y, Wahyuniati N, Anwar S, Yusri F, Haryanti N, Wijayanti NP, Rizal R, Fitriani D et al (2020). Parents' hesitancy towards vaccination in Indonesia: A cross-sectional study in Indonesia. Vaccine.

vii. Huang C, Wang Y, Li X, Ren L, Zhao J, Hu Y, Zhang L, Fan G, Xu J, Gu X et al (2019). Clinical features of patients infected with novel coronavirus in Wuhan, China. Lancet 2020, 395(10223):497-506.

viii. Chu DKW, Pan Y, Cheng SMS, Hui KPY, Krishnan P, Liu Y, Ng DYM, Wan CKC, Yang P, Wang Q et al (2020). Molecular Diagnosis of a Novel Coronavirus (2019-nCoV) Causing an Outbreak of Pneumonia. Clin Chem.

ix. Global Surveillance for human infection with novel coronavirus (2019-nCoV). In. (2020). World Health Organization.

x. Liu Y, Gayle A, Wilder-Smith A, J. R (2020). The reproductive number of COVID-19 is higher compared to SARS coronavirus. J Travel Med, https://doi.org/10.1093/jtm/taaa021

xi. Mahase E (2020). Coronavirus covid-19 has killed more people than SARSand MERS combined, despite lower case fatality rate. BMJ,368:m641.

xii. Chan JFW, Lau SKP, To KKW, Cheng VCC, Woo PCY, Yuen K-Y (2015). Middle East respiratory syndrome coronavirus: another zoonotic betacoronavirus causing SARS-like disease. Clinical microbiology reviews, 28(2):465-522.

xiii. Wang C, Horby PW, Hayden FG, Gao GF (2020). A novel coronavirus outbreak of global health concern. The Lancet.

xiv. Paraskevis D, Kostaki EG, Magiorkinis G, Panayiotakopoulos G, Sourvinos G, Tsiodras S (2020). Full-genome evolutionary analysis of the novel corona virus (2019-nCoV) rejects the hypothesis of emergence as a result of a recent recombination event. Infection, Genetics and Evolution 104212.

xv. Randhawa GS, Soltysiak MPM, El Roz H, De Souza CP, Hill KA, Kari L (2020). Machine learning analysis of genomic signatures provides evidence of associations between Wuhan 2019-nCoV and bat betacoronaviruses. BioRxiv.

xvi. Ji W, Wang W, Zhao X, Zai J, Li X (2020). Homologous recombination within the spike glycoprotein of the newly identified coronavirus may boost cross-species transmission from snake to human. Journal of medical virology.

xvii. David L Heymann \& Nahoko Shindo (2020). COVID-19: what is next for public health? THE LANCET.

xviii. A. Jamal, D. D. AL Narayanasamy, N. Q. Mohd Zaki and R. A. Abbas Helmi, (2019): Large Hall Temperature Monitoring Portal, in 2019 IEEE International Conference on Automatic Control and Intelligent Systems (I2CACIS), Selangor, Malaysia, pp. 62-67.

xix. Sim Liew Fong, David Chin Wui Yung, Falah YH Ahmed, Arshad Jamal, (2019). Smart City Bus Application with Quick Response (QR) Code Payment, in ICSCA '19 Proceedings of the 2019 8th International Conference on Software and Computer Applications, Pages 248-252, Penang, Malaysia - February 19 - 21.

xx. Viola P. and M. Jones (2001). Rapid Object Detection using a Boosted Cascade of Simple Features, Proc. 2001 IEEE Comput. Soc. Conf. Comput. Vis. Pattern Recognition. CVPR 2001, pp. 511-518.

xxi. Gonzalez-Lozoya, S.M., de la Calleja, J., Pellegrin, L. et al.(2020). Recognition of facial expression based on CNN features. Multimedia tool Applicaton 79, 13987-14007. https://doi.org/10.1007/s11042-020-08681-4 